J. Lake Sci.(湖泊科学), 2009, 21(2): 207-214

http://www.jlakes.org. E-mail: jlakes@niglas.ac.cn

(C2009 by Journal of Lake Sciences

\title{
基于水体反射光谱和组分浓度的固有光学特性反演算法*
}

\author{
杨 伟 ${ }^{1,2}$, 松下文经 ${ }^{2}$, 陈 晋 $1^{* *}$ \\ (1: 北京师范大学资源学院, 环境演变与自然灾害教育部重点实验室, 北京 100875) \\ (2: 日本筑波大学地球科学系, 筑波 305-8572, 日本)
}

摘 要: 吸收系数和后向散射系数是水体的固有光学特性参数, 在探测水体中各组分浓度的过程中起着至关重要的作用. 但 通常组分固有光学特性的测定是一个操作复杂且费时费力的过程. 提出了一种利用已知组分浓度和相应的反射光谱的水体样 本来推定水体中各组分的吸收和后向散射系数的算法, 并利用生物光学模型产生的模拟光谱数据对算法的合理性进行了验证. 结果表明, 在理想的实验条件下 (反射光谱满足机理模型, 且选取的训练样本相互独立), 可以高精度地反演出各组分的固有 光学特性, 从而证实了本算法在理论上的合理性.

关键词: 后向散射系数; 吸收系数; 反射光谱; 组分浓度

\section{Deriving inherent optical properties through training samples with known concentration of water constituents and reflectance spectra}

\author{
YANG Wei ${ }^{1,2}$, Matsushita Bunkei ${ }^{1} \&$ CHEN Jin ${ }^{1}$ \\ (1: Key Laboratory of Environment Change and Natural Disaster, Ministry of Education of China, College of Resource Science and \\ Technology, Beijing Normal University, Beijing 100875, P.R.China) \\ (2: Department of Geography, Tsukuba University, Tsukuba 305-8572, Japan)
}

Abstract: Coefficients of absorption and backscattering are inherent optical properties (IOPs) of water, which play extraordinarily important roles in the detection of water constituent concentrations. However, it is very complicate and time-consuming to measure the IOPs of waters. In this study, a novel algorithm was proposed to derive the IOPs of water constituents through training samples with measured constituents' concentrations and reflectance spectra. Simulated data based on bio-optical model was used to assess the proposed algorithm. Results demonstrated that when the spectra of samples satisfied the analytical model and the training samples were independent each other, the performance of the algorithm can be accepted, which indicates that the algorithm proposed in this paper is theoretically reasonable.

Keywords: Backscattering coefficient; absorption coefficient; reflectance spectra; concentration of water constituents

内陆湖泊水体在人们的日常生活和社会经济发展中发挥着至关重要的作用 ${ }^{[1]}$, 但全球湖泊水体富营 养化问题却日益严重. 传统的水质检测方法虽然有技术成熟、测量精度高的优点，但该方法费时费力，且 难以进行大规模的实时监测. 遥感技术的发展为进行实时快速、大范围的水质监测提供了有力的工具.

从遥感科学的角度, 水体可以分为一类水体和二类水体 ${ }^{[2]}$. 一类水体的光学特性主要取决于悬浮藻 类(如开阔海域), 而二类水体中除悬浮藻类之外, 还包含非藻类悬浮物质、黄色物质等等, 且它们的浓度 与叶绿素浓度并无明显相关. 近海岸水体和内陆湖泊均属于二类水体 ${ }^{[3]}$. 由于二类水体中悬浮藻类、非藻 类悬浮物质、黄色物质和水分子之间复杂的相互光学作用, 使得到目前为止遥感方法在二类水体的水质 监测中的应用尚不十分成功 ${ }^{[4]}$. 现有的利用光学遥感估测水质参数的方法通常有 3 种, 分别为经验方法、

* 国家自然科学基金(40871162)资助. 2008-11-03 收稿; 2008-12-03 收修改稿. 杨伟, 男, 1983 年生, 博士研究生.

** 通讯作者; E-mail: chenjin@ires.cn. 
半经验方法和机理模型方法 ${ }^{[5]}$. 其中, 机理模型方法以水体内光学传输的机理为理论基础, 是水质遥感 监测的一种重要方法 ${ }^{[6-7]}$. 但机理模型需要对水体组分的固有光学特性进行测量, 算法的操作难度较大, 这在一定程度上限制了该方法的广泛应用.

水体的固有光学特性参数包括吸收系数和后向散射系数. 为克服水体组分的吸收和后向散射系数难 于获取的问题, 近来人们提出了多种利用反射光谱推算固有光学特性参数的算法, 包括经验方法和光谱 最优化方法 ${ }^{[8-9]}$. 经验方法虽然简单易行, 但由于其物理意义不明确以及回归样本的局限性, 在多区域、 多时相的情形下并不适用; 光谱最优化方法基于水体的辐射传输过程, 具有精度高、适用范围广的优点, 但计算过程相当费时, 在真实卫星数据中难于应用, 且其结果依赖于高精度的光谱模型 ${ }^{[10]}$. 考虑到已有 算法存在的问题, 本文提出了一种基于生物光学模型, 利用已知组分浓度和相应的反射光谱的水体样本 来推定水体中各组分后向散射系数和吸收系数的算法, 并利用模拟数据对该算法的在理论上的合理性进 行了验证. 该算法既简单易操作, 又具有物理意义明确、反演精度高的特点.

\section{1 生物光学模型}

将水体的生物特性和光学特性关联起来的生物光学模型可以表示为 ${ }^{[1]}$ :

$$
R(0-, \lambda)=f u(\lambda), \quad u(\lambda)=b_{\mathrm{b}}(\lambda) /\left(a(\lambda)+b_{\mathrm{b}}(\lambda)\right)
$$

其中, $R(0-, \lambda)$ 是刚好在水面以下的辐照度反射率, $a$ 是水体总吸收系数, $b_{\mathrm{b}}$ 是水体总后向散射系数, $f$ 因 子取决于水体的散射特性及周围光场的几何结构. 根据朗伯体假定, $f$ 因子可以表示为 ${ }^{[12]}$ :

$$
f \approx \pi(0.0949+0.0794 u(\lambda))
$$

需要说明的是, 这个模型没有考虑叶绿素的苂光效应及水体的拉曼散射.

水体的固有光学特性可以表示为水体中各个组分的贡献与水分子的光学特性的线性和. 对于二类水 体, 通常需要考虑 3 种组分: 藻类(即 Chl.a)、非藻类悬浮物 (Non-phytoplankton Suspended Sediments, NPSS) 和黄色物质(Colored Dissolved Organic Matter, CDOM). 于是, 水体的总吸收系数和总后向散射系数可以 分别表示为:

$$
\begin{aligned}
& a(\lambda)=a_{\mathrm{w}}(\lambda)+C h l . a \times a_{\mathrm{p}}^{*}(\lambda)+\operatorname{Tr} \times a_{\mathrm{n}}^{*}(\lambda)+a_{\mathrm{CDOM}}(\lambda) \\
& b_{\mathrm{b}}(\lambda)=b_{\mathrm{b}, \mathrm{w}}(\lambda)+C h l . a \times b_{\mathrm{b}, \mathrm{p}}^{*}(\lambda)+\operatorname{Tr} \times b_{\mathrm{b}, \mathrm{n}}^{*}(\lambda)
\end{aligned}
$$

其中, $a_{\mathrm{w}}$ 和 $b_{\mathrm{b}, \mathrm{w}}$ 分别为水分子的吸收和后散射系数, $a_{\mathrm{b}, \mathrm{p}}^{*}$ 和 $a_{\mathrm{b}, \mathrm{n}}^{*}$ 分别为 Chl.a 和 NPSS 的单位浓度吸收系数, $b_{\mathrm{b}, \mathrm{p}}^{*}$ 和 $b_{\mathrm{b}, \mathrm{n}}^{*}$ 分别为 Chl.a 和 NPSS 的单位浓度后向散射系数. CDOM 的吸收系数为:

$$
a_{\mathrm{CDOM}}(\lambda)=a_{\mathrm{CDOM}}(400) \exp [-S(\lambda-400)]
$$

其中, $a_{\mathrm{CDOM}}(400)$ 为 $\mathrm{CDOM}$ 在 $400 \mathrm{~nm}$ 处的吸收系数. 利用 Brando 和 Dekker 测定的二类水体各组分的固 有光学特性(图 1) ${ }^{[13]}$, 通过基于生物光学模型的模拟数据来进行算法开发和精度检验.

\section{2 固有光学特性反演算法的开发}

从式(3)可以得出, 水体中 Chl.a 和 NPSS 的后向散射和吸收系数均与其浓度成正比, 具体可以表示 为:

$$
\begin{aligned}
& \frac{a_{\mathrm{p} 1}(\lambda)}{a_{\mathrm{p} 2}(\lambda)}=\frac{b_{\mathrm{bp} 1}(\lambda)}{b_{\mathrm{bp} 2}(\lambda)}=\frac{[\text { Chl.a }]_{1}}{[\text { Chl.a }]_{2}} \\
& \frac{a_{\mathrm{n} 1}(\lambda)}{a_{\mathrm{n} 2}(\lambda)}=\frac{b_{\mathrm{bn} 1}(\lambda)}{b_{\mathrm{bn} 2}(\lambda)}=\frac{[N P S S]_{1}}{[N P S S]_{2}}
\end{aligned}
$$


其中, $a_{\mathrm{p} 1,} b_{\mathrm{bp} 1}$ 和 $a_{\mathrm{p} 2,}, b_{\mathrm{bp} 2}$ 分别对应于浓度为 $[C h l . a]_{1}$ 和 $[C h l . a]_{2}$ 的藻类的吸收和后向散射 系数, $a_{\mathrm{n} 1,}, b_{\mathrm{bn} 1}$ 和 $a_{\mathrm{n} 2}, b_{\mathrm{bn} 2}$ 分别对应于浓度为 $[N P S S]_{1}$ 和 $[N P S S]_{2}$ 的非藻类悬浮物的吸收和 后向散射系数. 从公式(4)可知, CDOM 在各 波段的吸收系数正比于其在 $400 \mathrm{~nm}$ 处的吸收 系数:

$$
\frac{a_{\mathrm{CDOM} 1}(\lambda)}{a_{\mathrm{CDOM} 2}(\lambda)}=\frac{\left[a_{\mathrm{CDOM}}(400)\right]_{1}}{\left[a_{\mathrm{CDOM}}(400)\right]_{2}}
$$

公式(1)中的变量 $u$ 可以由反射光谱简单地 得到，也可以表示为:

$$
u=\frac{b_{\mathrm{bw}}+b_{\mathrm{bp}}+b_{\mathrm{bn}}}{a_{\mathrm{w}}+a_{\mathrm{p}}+a_{\mathrm{c}}+a_{\mathrm{n}}+b_{\mathrm{bw}}+b_{\mathrm{bp}}+b_{\mathrm{bn}}}
$$

将上式的分子、分母同时除以水分子的后 项散射系数, 并进一步变换为:

$$
\begin{aligned}
& (1-u) \frac{b_{\mathrm{bp}}}{b_{\mathrm{bw}}}+(1-u) \frac{b_{\mathrm{bn}}}{b_{\mathrm{bw}}}-u \frac{a_{\mathrm{w}}}{b_{\mathrm{bw}}}-u \frac{a_{\mathrm{p}}}{b_{\mathrm{bw}}}-u \frac{a_{\mathrm{n}}}{b_{\mathrm{bw}}} \\
& -u \frac{a_{\mathrm{c}}}{b_{\mathrm{bw}}}=u-1
\end{aligned}
$$

为推算各组分的吸收和后向散射系数, 我 们假定有一组已知各组分浓度和反射光谱的水 体样本(设共有 $N$ 个, 下文称作“训练样本”). 从其中任意选取一个样本作为参考样本, 其 Chl.a 和 NPSS 的浓度分别记为 $[C H L-a]^{\prime}$ 和
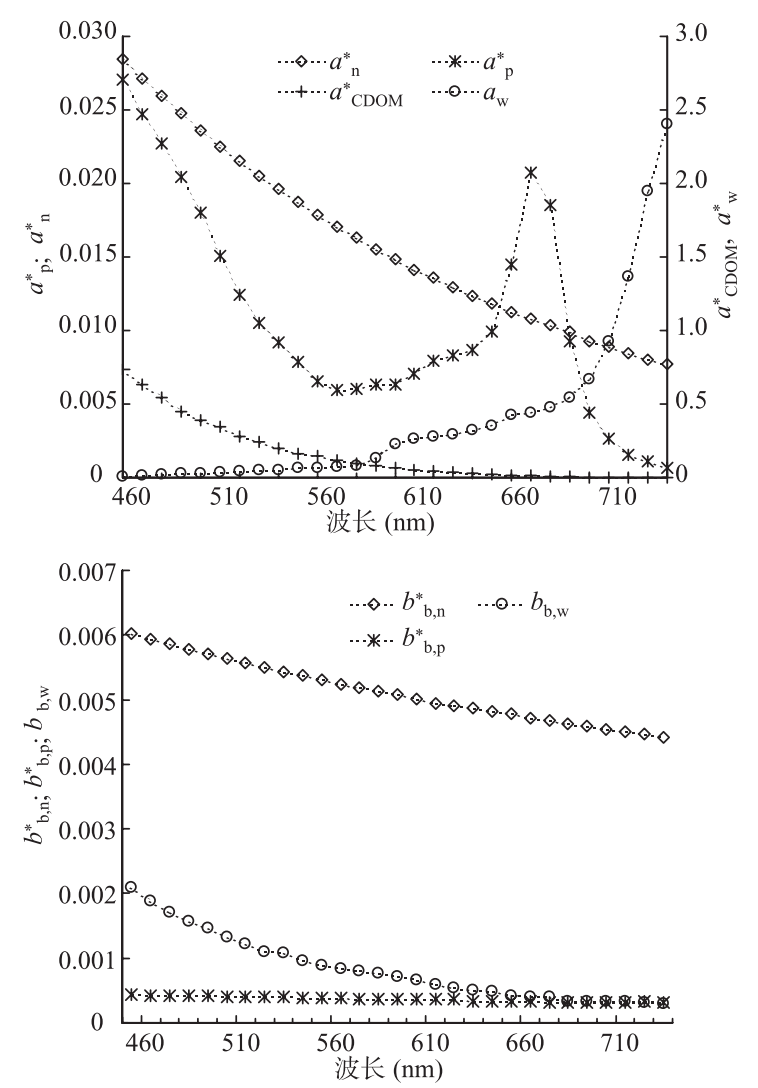

图 1 单位浓度水体组分的固有光学特性参数 ${ }^{[13]}$ $\left(a_{\mathrm{p}}^{*}\left(\mathrm{~m}^{2} / \mu \mathrm{g}\right), b_{\mathrm{b}, \mathrm{p}}^{*}\left(\mathrm{~m}^{2} / \mu \mathrm{g}\right), a_{\mathrm{n}}^{*}\left(\mathrm{~m}^{2} / \mathrm{mg}\right), b_{\mathrm{b}, \mathrm{n}}^{*}\right.$ $\left(\mathrm{m}^{2} / \mathrm{mg}\right), a_{\mathrm{w}}\left(\mathrm{m}^{-1}\right), b_{\mathrm{b}, \mathrm{w}}\left(\mathrm{m}^{-1}\right), a_{\text {CDOM: }}^{*}$ 无量纲 $)$

Fig.1 Specific inherent optical properties for water constituents [NPSS]', CDOM 在 $400 \mathrm{~nm}$ 的吸收记为 $\left[a_{\mathrm{CDOM}}(400)\right]^{\prime}$. 利用公式 $(5)-(7)$, 其余 $N-1$ 个训练样本的吸收和后向散射系数均可以用该参考样本的吸 收和后向散射系数表达, 从而公式(9)可以写作:

$$
\begin{array}{ccc}
\frac{[C H L-a]_{i}}{[C H L-a]^{\prime}}\left(1-u_{i}\right) \frac{b_{\mathrm{bp}}^{\prime}}{b_{\mathrm{bw}}}+\frac{[N P S S]_{i}}{[N P S S]^{\prime}}\left(1-u_{i}\right) \frac{b_{\mathrm{bn}}^{\prime}}{b_{\mathrm{bw}}}-u_{i} \frac{a_{\mathrm{w}}}{b_{\mathrm{bw}}}-\frac{[C H L-a]_{i}}{[C H L-a]^{\prime}} u_{i} \frac{a_{\mathrm{p}}^{\prime}}{b_{\mathrm{bw}}}-\frac{[N P S S]_{i}}{[N P S S]^{\prime}} u_{i} \frac{a_{\mathrm{n}}^{\prime}}{b_{\mathrm{bw}}} & \\
- & -\frac{\left[a_{\mathrm{CDOM}}(400)\right]_{i}}{\left[a_{\mathrm{CDOM}}(400)\right]^{\prime}} u_{i} \frac{a_{\mathrm{CDDM}}^{\prime}}{b_{\mathrm{bw}}}=u_{i}-1, & i=1, \cdots \cdots N
\end{array}
$$

其中, $b_{\mathrm{bp}}^{\prime}$ 和 $b_{\mathrm{np}}^{\prime}$ 分别为参考样本中 Chl. $a$ 和 NPSS 的后向散射, $a_{\mathrm{p}}^{\prime} 、 a_{\mathrm{n}}^{\prime}$ 和 $a_{\mathrm{CDOM}}^{\prime}$ 为参考样本中 Chl.a、NPSS 和 CDOM 的吸收系数.

由于共有 $N$ 个训练样本, 于是可以列出共 $N$ 个如上的方程. 在这 $N$ 个方程中, 变量 $b_{\mathrm{bp}}^{\prime} / b_{\mathrm{bw}}, b_{\mathrm{bn}}^{\prime} / b_{\mathrm{bw}}$, $a_{\mathrm{w}} / b_{\mathrm{bw}}, a_{\mathrm{p}}^{\prime} / b_{\mathrm{bw}}, a_{\mathrm{n}}^{\prime} / b_{\mathrm{bw}}$ 和 $a_{\mathrm{CDOM}}^{\prime} / b_{\mathrm{bw}}$ 为公共未知量. 若独立方程的个数大于或等于未知量的个数, 就可以 求解出这 6 个未知量. 进一步将方程(10)的解分别除以对应的组分浓度并乘以水分子后向散射系数就可 以得到水体各组分的单位浓度吸收系数和后向散射系数. 


\section{3 结果分析}

本文利用生物光学模型产生模拟反射光谱数据的方式来检验反演算法的精度. 采用最少训练样本数 量进行光学特性参数的推定, 即只使用 6 个训练样本. 水体中各组分的浓度来自均匀分布随机数发生器, 产生方式为: Chl.a 和 NPSS 的浓度分别为 $0-120 \mu \mathrm{g} / \mathrm{m}^{3}$ 和 $0-100 \mathrm{mg} / \mathrm{m}^{3}$ 范围内的均匀随机数, CDOM 在

表 1 训练样本水体组分浓度

Tab.1 Concentrations of water constituents within the training samples

\begin{tabular}{llll}
\hline & Chl.a $\left(\mu \mathrm{g} / \mathrm{m}^{3}\right)$ & NPSS $\left(\mathrm{mg} / \mathrm{m}^{3}\right)$ & $a_{\mathrm{CDOM}}(400)\left(\mathrm{m}^{-1}\right)$ \\
\hline S1 & 107.75 & 10.99 & 3.09 \\
S2 & 66.99 & 63.19 & 14.24 \\
S3 & 92.03 & 34.21 & 10.41 \\
S4 & 70.69 & 5.95 & 2.31 \\
S5 & 7.24 & 55.19 & 8.47 \\
S6 & 116.77 & 44.88 & 11.03 \\
\hline
\end{tabular}

$400 \mathrm{~nm}$ 处的吸收系数为 $0-15 \mathrm{~m}^{-1}$ 范围内的均匀 随机数(表 1).

训练样本对应的反射光谱如图 2 所示. 从 图中可以看出, 所产生的训练样本既含有 Chl.a 的典型光谱特性, 即在绿光波段的反射 峰和红光波段的吸收谷, 如 $S 1$ 和 $S 4$; 也反应了 NPSS 在各波段反射率较高的特性, 如 $S 5$; 而 CDOM 的吸收特性则使得各个样本的反射率 下降. 通过检验如公式(10)所示的 6 个方程可 知, 这组训练样本之间相互独立, 可以用于推 定水体固有光学特性参数.

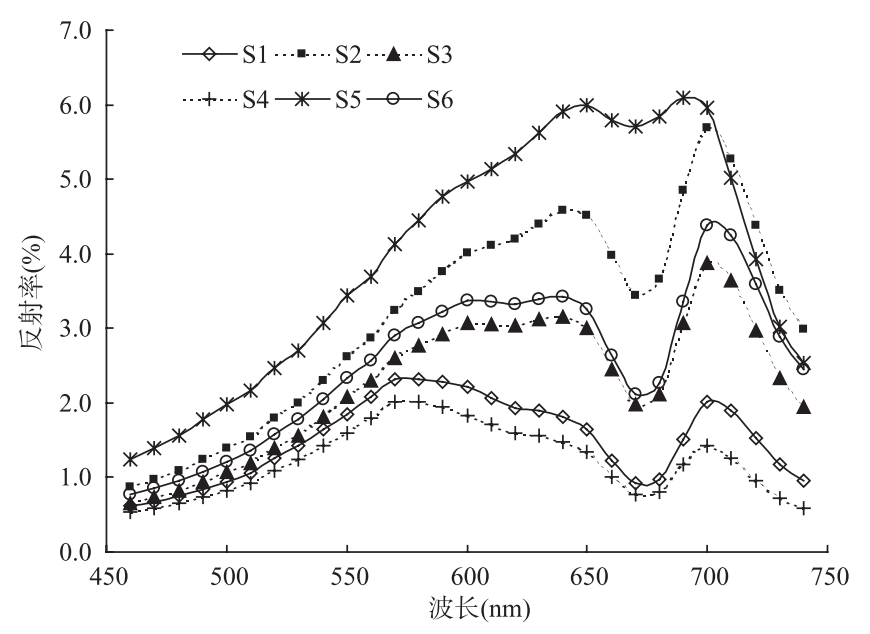

图 2 训练样本的反射光谱

Fig.2 The reflectance spectra of training samples

由于本算法将水分子后项散射系数视作已知参数, 所以最终可以得到水体组分的 6 个固有光学特性参数, 包括水分子的吸收系数 $\left(a_{\mathrm{w}}\right), \mathrm{Chl} . \mathrm{a}$ 和 NPSS 的单位浓度吸收系数和后项散射系数 $\left(a_{\mathrm{p}}^{*}, a_{\mathrm{n}}{ }^{*}, b_{\mathrm{b}, \mathrm{p}}, b_{\mathrm{b}, \mathrm{n}}\right), \mathrm{CDOM}$ 在 $400 \mathrm{~nm}$ 处的吸收系数 $\left(a_{\mathrm{CDOM}}{ }^{*}\right.$. 各参数的推定值和真实值的散点图如图 3 所示. 从图中可知, 数据点几乎完全 分布在“ $1: 1$ ”线上, 而其回归方程的决定系数 $\left(R^{2}\right)$ 均为 “ 1.0 ” , 斜率和截距分别十分接近 “1.0” 和 “ 0 ” (表 2). 为 定量评价反演结果的误差, 本文采用相对均方根误差(Relative RMSE)作为反演误差的定量指标:

$$
\text { Relative } R M S E=\sqrt{\frac{\sum_{i=1}^{N}\left(X_{i}-Y_{i}\right)^{2}}{N}} /\left(\sum_{i=1}^{N} X_{i} / N\right)
$$

式中, $N$ 为光学特性参数的波段数目, $X_{i}$ 为真实光学参数, $Y_{i}$ 为反演所得的光学参数. 各个固有光学特性参 数对应的相对均方根误差如表 2 所示. 从表可知各参数的 Relative RMSE 均十分接近于 “ 0 ”, 即反演误差 几乎为 “ 0 ” . 所有这些指标均表明, 本算法利用如上的训练样本的反演结果精度是十分理想的, 从而很 好地证实了本算法在理论上的合理性. 
表 2 反演结果精度评价指标

Tab.2 Index for evaluating the performance of algorithm

\begin{tabular}{ccccccc}
\hline & $b_{\mathrm{b}, \mathrm{p}}^{*}$ & $b_{\mathrm{b}, \mathrm{n}}^{*}$ & $a_{\mathrm{W}}$ & $a_{\mathrm{p}}^{*}$ & $a_{\mathrm{n}}^{*}$ & $a_{\mathrm{CDOM}}^{*}$ \\
\hline Relative RMSE & $4.22 \mathrm{E}-04$ & $4.12 \mathrm{E}-04$ & $7.42 \mathrm{E}-04$ & $6.05 \mathrm{E}-04$ & $5.16 \mathrm{E}-04$ & $8.82 \mathrm{E}-04$ \\
决定系数 & 1.00 & 1.00 & 1.00 & 1.00 & 1.00 & 1.00 \\
斜率 & 0.99 & 0.99 & 1.00 & 1.00 & 0.99 & 0.99 \\
截距 & $4.00 \mathrm{E}-07$ & $6.00 \mathrm{E}-06$ & $-3.00 \mathrm{E}-05$ & $-1.00 \mathrm{E}-08$ & $3.00 \mathrm{E}-06$ & $8.00 \mathrm{E}-06$ \\
\hline
\end{tabular}
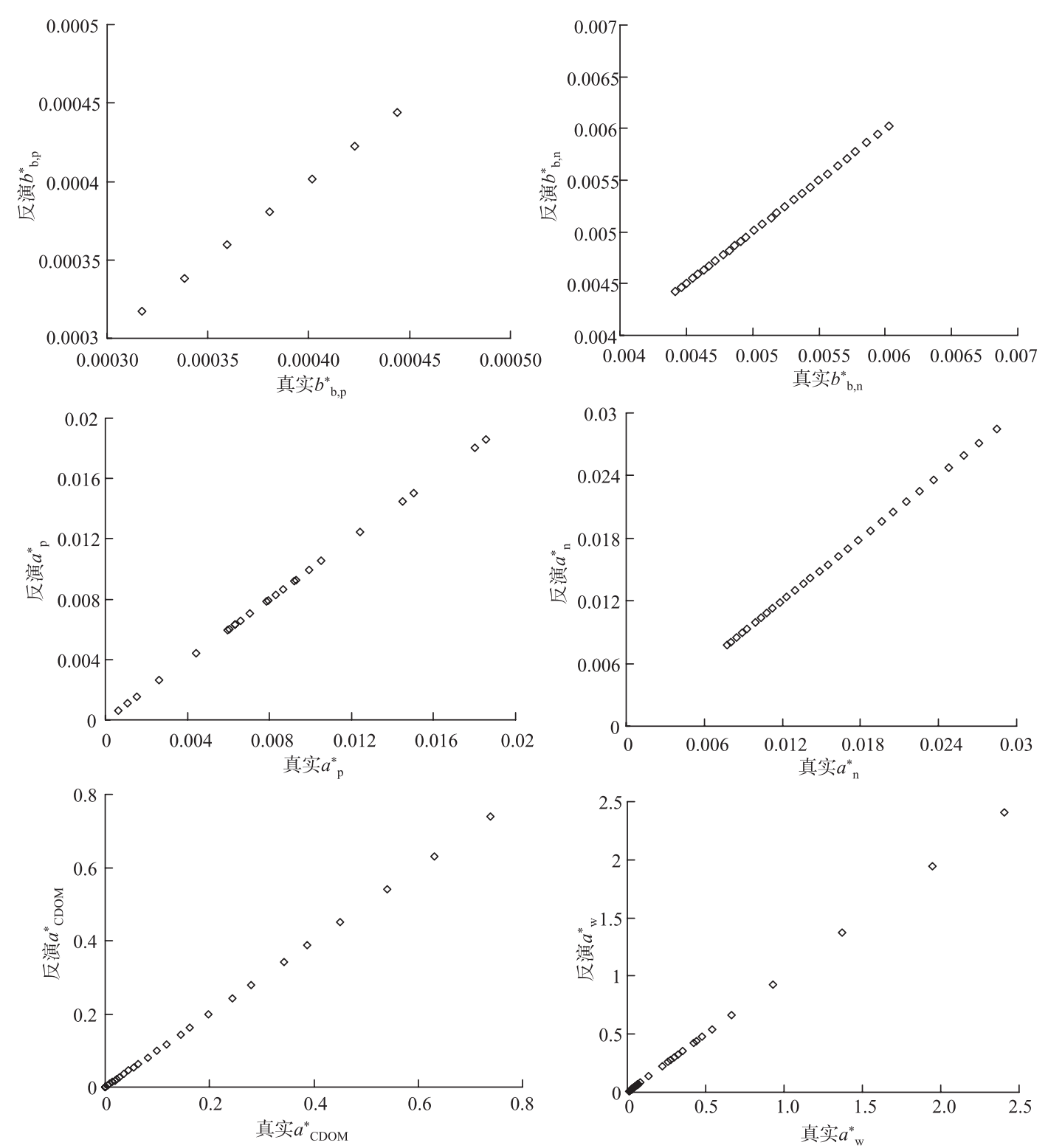

图 3 反演单位浓度固有光学特性参数与真实参数的散点图

Fig.3 Scatter plots of retrieved SIOPs and truth data

为检验反演算法的稳定性, 将如上的模拟重复进行了 30 次 (即共 30 组不同浓度的训练样本), 其反演 
结果对应的 $R^{2}$ 和 Relative RMSE 如图 4 所示. 结果表明对于大多数训练样本的反演效果仍十分理想, $R^{2}$ 和 Relative RMSE 分别接近 “ $1.0 ”$ 和 “ 0 ”. 但有少数组别, 其反演精度较低. 如第 11 组训练样本得到的 Chl.a 和 NPSS 的后向散射系数和水分子的吸收系数均出现了较大的误差. 其对应的反射光谱和组分浓度分别如 图 5 和表 3 所示. 通过方程组(10)的检验可知, 第 11 组中的训练样本相关性过高, 从而导致了光学参数反演 结果的显著误差. 这表明, 训练样本间的相关性对反演结果的精度起着至关重要的作用. 所以, 在选取训练 样本时必须使用方程组(10)对样本相关性进行检验, 只有相互独立的训练样本才能够人选.
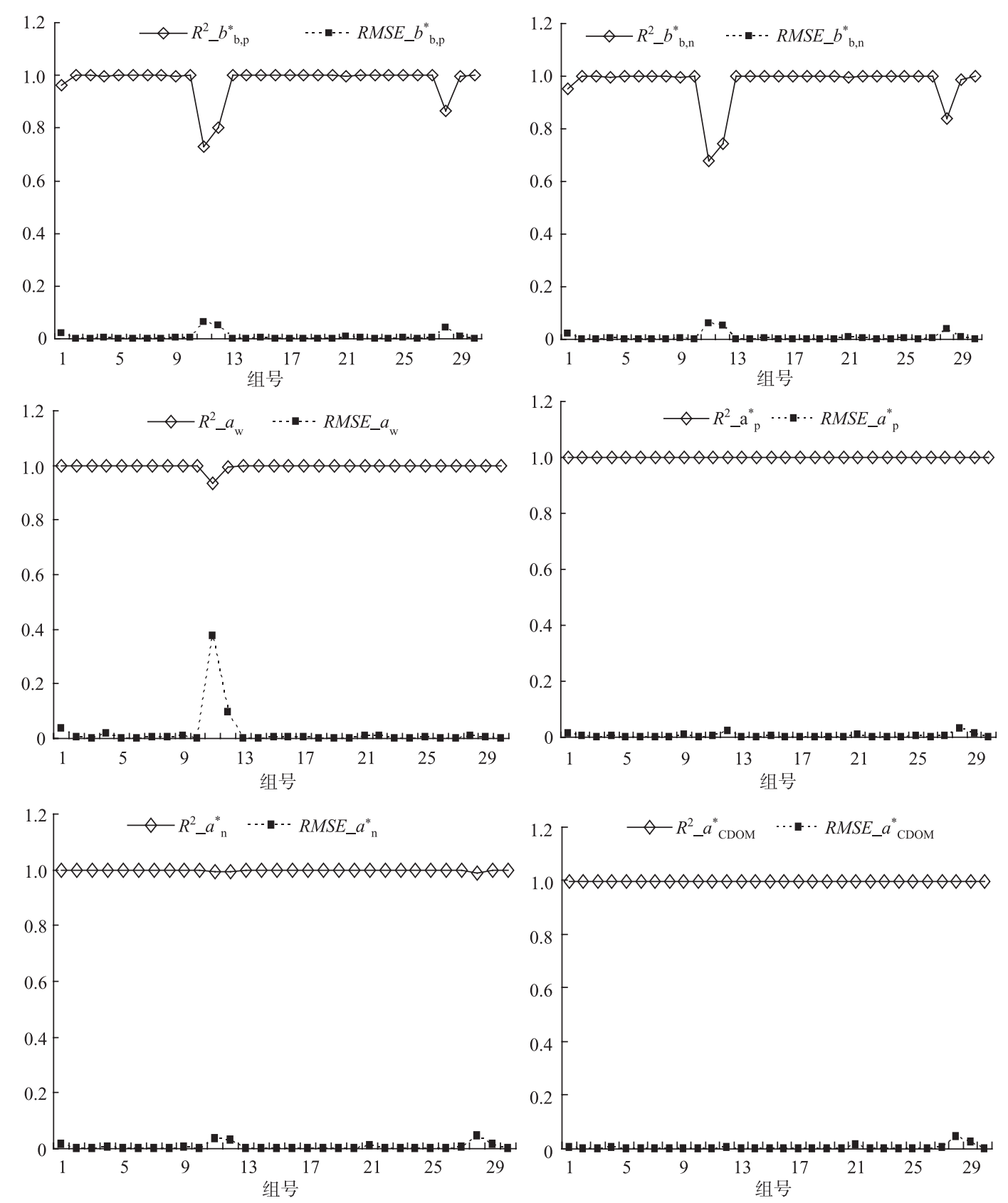

图 430 组模拟数据的反演结果对应的 $R^{2}$ 和相对 $R M S E$

Fig. $4 R^{2}$ and relative $R M S E$ corresponding to the 30 groups of simulated data 


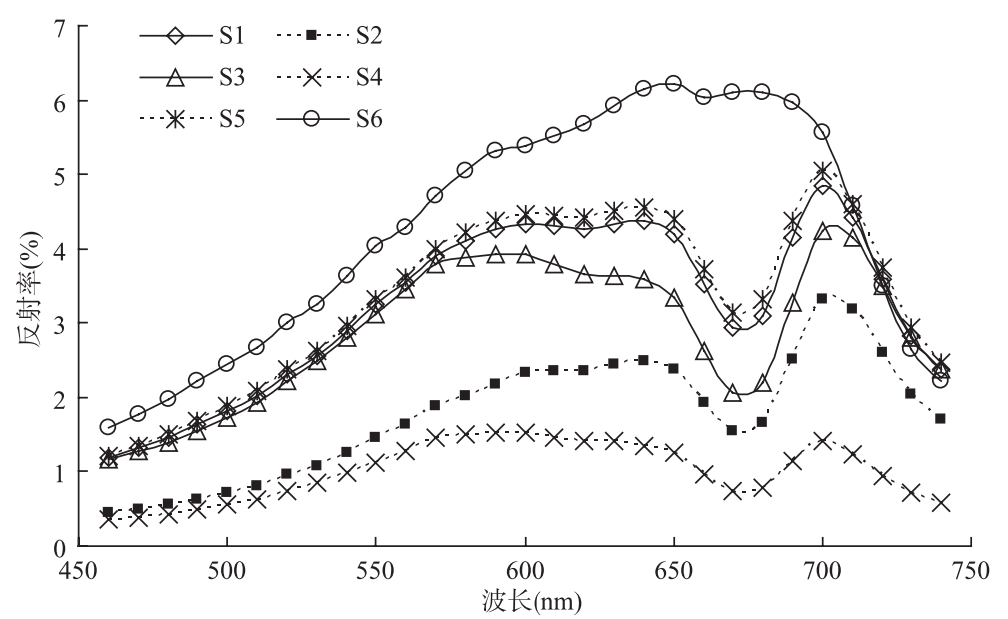

图 5 相关性过高的训练样本光谱(不适于推算 SIOPs)

Fig.5 Spectra of training samples with high correlation (inappropriate for deriving SIOPs)

\section{4 结论与讨论}

在生物光学模型的基础上, 本文提出了 一种利用已知组分浓度和相应的反射光谱的 训练样本反演水体固有光学特性参数的算法. 在反射光谱数据满足生物光学模型且训练样 本相互独立的理想实验条件下，该算法的反 演结果准确可靠，从而证实了该算法在理论 上的合理性. 本文的算法基于水体的机理模 型，具有明确的物理意义，其结果可以在多 区域、多时相的情形下应用. 相对于水体组 分的吸收系数和后向散射系数, 组分浓度和 反射光谱是两个更易于测定的指标. 本文的算法利用组分浓度和反射光谱得到了水体各组分的吸收和后 向散射系数, 这在一定程度上可以降低一些以固有光学特性参数为输人数据的水体组分浓度反演算法的操 作难度, 如矩阵反演法(Matrix Inversion Method) ${ }^{[13]}$, 光谱分解算法(Spectral Decomposition Approach) ${ }^{[14-15]}$ 等等.

本文的研究重点在于算法的理论研究, 所以对算法的真实数据验证和实际卫星数据的应用尚未进行. 本算法基于水体的反射率来推导固有光学特性参数，而卫星数据可以提供水体的反射率，所以本算法可 以应用到真实卫星数据中. 这些工作将在下一步的研究中完成. 在使用本算法推定水体固有光学特性时, 仅需要在卫星过境时采集满足算法要求的水体样本, 确定其组分浓度值即可. 而各组分浓度, 如叶绿素, 总悬浮物的浓度及 CDOM 在 $400 \mathrm{~nm}$ 处的吸收都是水质监测的常规参数, 从既有的测量数据即可得到. 再 结合以往的卫星数据，即可以利用本算法推定过去的水体固有光学特性，为以往的水质分析提供有用的 数据. 另外, 由于本算法是基于机理模型的, 所以一次准确的测量即可以用于多时相、多区域(假定水体 的固有光学特性一致)的水质参数探测中, 从而可以大大降低野外现地观测的操作难度和工作强度.

\section{5 参考文献}

[1] Giardino C, Pepe M, Brivio PA et al. Detecting chlorophyll, Secchi disk depth and surface temperature in a sub-alpine lake using Landsat imagery. Science of the Total Environment, 2002, 68: 19-29. 
[2] Morel A, Prieur L. Analysis of variations in ocean color. Limnology and Oceanography, 1977, 22: 709-722.

[3] Gin KYH, Koh ST, Lin II. Spectral irradiance profile of suspended marine clay for the estimation of suspended sediment concentration in tropical waters. International Journal of Remote Sensing, 2003, 24: 3235-3245.

[4] Gin KYH, Koh ST, Lin II. Study of the effect of suspended marine clay on the reflectance spectra of phytoplankton. International Journal of Remote Sensing, 2002, 23: 2163-2178.

[5] 李素菊, 王学军. 内陆水体水质参数光谱特征与定量遥感. 地理学与国土研究, 2002, (2): 26-29.

[6] Haltrin VI. Self-consistent approach to the solution of the light transfer problem for irradiances in marine waters with arbitrary turbidity, depth, and surface illumination: I: case of absorption and elastic scattering. Applied Optics, 1998, 37(18): 3773-3784.

[7] Forget P, Broche P, Naudin JJ. Reflectance sensitivity to solid suspended sediment stratification in coastal water and inversion: a case study. Remote Sensing of Environment, 2001, 77: 92-103.

[8] Sathyendranath S, Cota G, Stuart V et al. Remote sensing of phytoplankton pigments: a comparison of empirical and theoretical approaches. International Journal of Remote Sensing, 2001, 22: 249-273.

[9] Lee ZP, Carder KL, Peacock TG et al. Method to derive ocean absorption coefficients from remote-sensing reflectance. Applied Optics, 1996, 35: 453-462.

[10] Lee Z, Carder KL, Hawes SK et al. Deriving inherent optical properties from water color: a multiband quasi-analytical algorithm for optically deep waters. Applied Optics, 2002, 41(27): 5755-5772.

[11] Gordon HR, Brown OB, Jacobs MM. Computed relationships between the inherent and apparent optical properties of a flat homogeneous ocean. Applied Optics, 1975, 14(2): 417-427.

[12] Gordon HR, Brown OB, Evens RH et al. A semianalytic radiance model of ocean color. Journal of Geophysical Research, 1988, 93D: 10909-10924.

[13] Brando VE, Dekker AG. Satellite hyperspectral remote sensing for estimating estuarine and coastal water quality. IEEE Transaction on Geoscience and Remote Sensing, 2003, 41(6): 1378-1387.

[14] Oyama Y, Matsushita B, Fukushima et al. A new algorithm for estimating chlorophyll-a concentration from multi-spectral satellite data in Case II waters: a simulation based on a controlled laboratory experiment. International Journal of Remote Sensing, 2007, 28: 1437-1453.

[15] Oyama Y, Matsushita B, Fukushima T et al. Application of spectral decomposition algorithm for mapping water quality in a turbid lake (Lake Kasumigaura, Japan) from Landsat TM data. ISPRS Journal of Photogrammetry \& Remote Sensing(doi: $10.1016 /$ j.isprsjprs.2008.04.005)(in press). 